\title{
Nano-size anodization of Si by using an atomic force microscope
}

\author{
N. Takahashi, S. Harako and X. Zhao \\ Department of Physics, Tokyo University of Science, 1-3 Kagurazaka, Shinjuku-ku, Tokyo 162-8601, Japan \\ Fax: 81-03-5228-8231, e-mail: xwzhao@rs.kagu.tus.ac.jp
}

\begin{abstract}
Nano meter-sized $\mathrm{SiO}_{2}$ lines have been formed on silicon (Si) surface by using AFM tip-induced anodization. A thin water film between the tip and the Si surface worked as a highly resistive electrolyte in a wet air atmosphere. The applied bias voltage dependence of widths and heights of the oxide lines was investigated. It was revealed that the Si was oxidized at very low bias, such as $2.2 \mathrm{~V}$. The thinnest line width of $100 \mathrm{~nm}$ has been achieved. The carrier type and concentration were also important factors on the size control in the anodic oxidation.
\end{abstract}

Key words: AFM, anodization, silicon, $\mathrm{SiO}_{2}$, nanostructure

\section{Introduction}

Recently, an atomic force microscope (AFM) takes an important role in surface Science [1]. It is mainly used for measuring the nanometer-sized structures in the material surfaces. J. A. Dagata proposed a new usage of AFM for an anodization of crystals in 1989 [2]. The anodization using AFM serves as a leading tool of the bottom-up nano-processing technology, which can form nanostructures at an arbitrary position. The AFM anodization has been also used in nanometer scale oxidization of semiconductors, such as silicon (Si) [3]. Recently, this technique has been used to form the insulator area in nanodevice fabrication process for observing Coulomb blockade characteristics [4, 5]. The line width of the oxide on the Si surface formed by AFM anodization is limited by the diameter of the cantilever top. Then a carbon nanotube tip was suggested to be a good candidate in AFM anodization [6]. It is expected that the AFM anodization will occupy an important position in nano processing technology in the future. It is still a far goal to fabricate a single electron transistor by AFM only because of the tunnel effect become observable in junction length less than $1 \mathrm{~nm}$. The theoretical analysis for the AFM anodization, on the other hand, is also not carried out yet. The applied bias voltage, scanning speed and the humidity were considerable factors affecting the AFM anodization $[7,8]$. The dependence of the carrier density and type of Si substrates are also important, but there is no enough data in literature [9].

In this paper, we present the formation results of nanolines and nanodots by using AFM tip-induced anodization. A thin water sheet between the tip and the $\mathrm{Si}$ surface behaved as the electrolyte. Theoretical analysis indicated that the Si was oxidized at very low biased voltages. Conductive types of the Si wafer also alternated the width and depth of the oxide region. A model of hole-assisted oxidation was used to explain the results.

\section{Theoretical analysis and experimental}

As a normal anodization process, chemical reactions at electrodes are key points in AFM induced anodization, too. Because the anodization used thin water layer on the surface as the electrolyte, electrolysis of the water and a motion of $\mathrm{H}^{+}$and $\mathrm{OH}^{-}$ions came to be important.
The AFM probe tip was used as a cathode and $\mathrm{Si}$ as an anode, respectively. In a conventional anodic oxidation process, electric double layers which are formed by separated $\mathrm{H}^{+}$and $\mathrm{OH}^{-}$ions by $1 \sim 2 \mathrm{~nm}$ exist on the surfaces of both electrodes. In contrast, the surface water layer in the AFM anodization is as thin as about $2 \mathrm{~nm}$, it works as the electric double layer in the process. The $\mathrm{H}_{2} \mathrm{O}$ separates to $\mathrm{H}^{+}$and $\mathrm{OH}^{-}$ions while applying a positive bias on $\mathrm{Si}$ anode. Figure 1 shows the band diagram of the AFM anodization for the case of p-type Si. As the conventional anodization, the energy of electrons is written by the voltage expression. Figure 1(a) shows a case of zero bias with the energy of generating $\mathrm{H}_{2}$ as the standard level $\mathrm{V}=0 \mathrm{~V}$. The Fermi levels of both electrodes are pinning at the redox energy of water, Eredox $=+0.62 \mathrm{~V}$, which is the intermediate value of the energy of electrolyzing water, i.e. $E=+1.23$ V. Although there is no $\mathrm{O}_{2}$ generation in this process, the above-mentioned energy is considered to be necessary to provide enough $\mathrm{H}^{+}$and $\mathrm{OH}^{-}$ions to continue the anodization. The energy positions of the conduction band bottom and valence band top of $\mathrm{Si}$ are $-0.51 \mathrm{~V}$ and $+0.61 \mathrm{~V}$, respectively. So that a hole-rich surface occurs which may promote the hole assisted oxidation. When applying a forward bias on the system, the $\mathrm{H}^{+}$moves to the AFM probe surface and receives an electron to generate $\mathrm{H}_{2}$, as shown in Fig. 2(b). Meanwhile, the $\mathrm{OH}^{-}$provides $\mathrm{O}$ to oxidize a backbond of a surface $\mathrm{Si}$ atom by replacing a hole. Then the $\mathrm{OH}$ dehydrogenates to $\mathrm{H}_{2} \mathrm{O}$. This cycle repeats to promote the anodization. Another possible process is that $\mathrm{H}^{+}$moves through the $\mathrm{SiO}_{2}$ layer and combines $\mathrm{OH}^{-}$to form $\mathrm{H}_{2} \mathrm{O}$ at the $\mathrm{SiO}_{2}$ surface. Nevertheless, the anodization goes on due to the applied bias voltage. The reactions in the water sheet and the electrodes are summarized as following,

Water sheet: $2 \mathrm{H}_{2} \mathrm{O} \rightarrow 2 \mathrm{H}^{+}+2 \mathrm{OH}^{-}$

$$
\begin{aligned}
& \text { Anode (Si): } \mathrm{Si}+2 \mathrm{OH}^{-}+2 \mathrm{~h}^{+} \rightarrow \\
& \qquad \mathrm{SiO}_{2}+2 \mathrm{H}^{+}+2 \mathrm{e}^{-}
\end{aligned}
$$

Cathode (tip): $2 \mathrm{H}^{+}+2 \mathrm{e}^{-} \rightarrow 2 \mathrm{H}_{2} \uparrow$ 

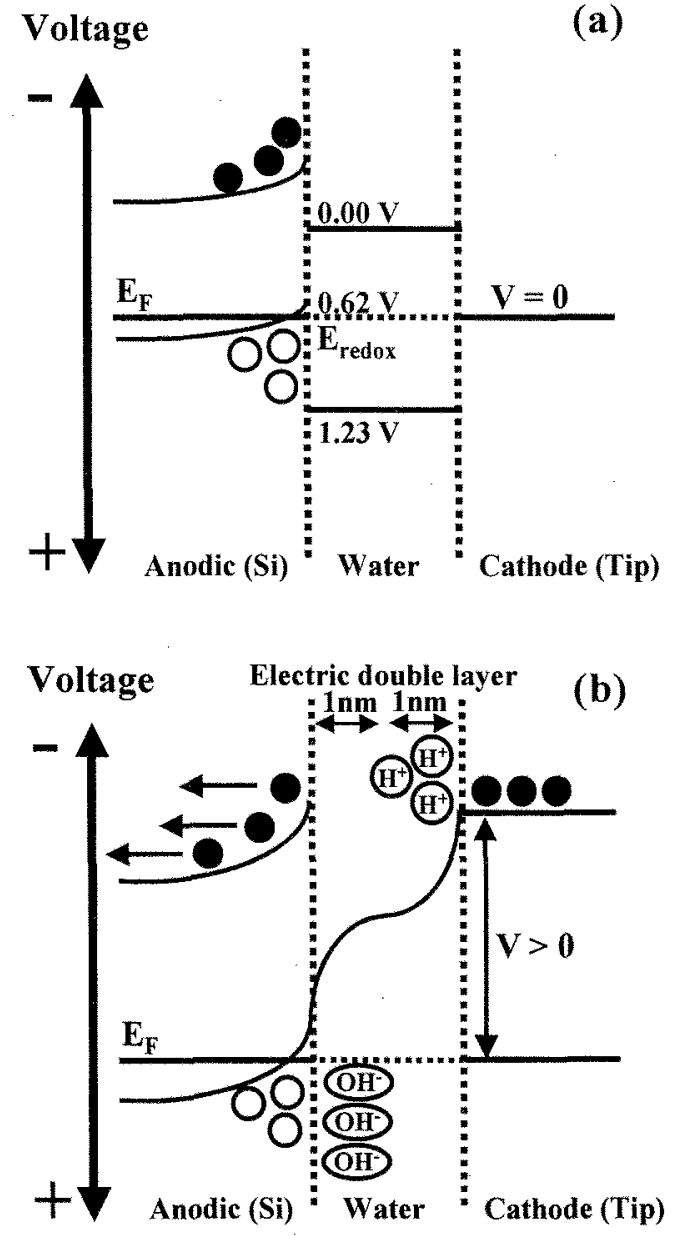

Fig. 1. Band diagrams of an atomic force microscope induced anodization of $\mathrm{p}-\mathrm{Si}(100)$ for the cases of (a) $\mathrm{V}=0$ and (b) $\mathrm{V}>0$.

The necessary potential for completing the above-mentioned reactions can be easily calculated based on thermo-chemical equations as $E=1.63 \mathrm{~V}$. Chemical reactions in the electrodes, however, did not occur while applying a bias voltage like this. Metals used as the electrode materials have their particular overvoltages to change electrons and generate $\mathrm{H}_{2}$. The AFM probe was a thin Rhodium ( $\mathrm{Rh}$ ) coated Si tip, where $\mathrm{H}_{2}$ gas was generated by $\mathrm{H}^{+}$ions combined with electrons. The overvoltage of $\mathrm{Rh}$ is $0.05 \mathrm{~V}$, as shown in Ref [11]. For the overvoltage of Si, which is the anode, there is no reported data. Overvoltage of $\mathrm{Si}$ was considered depending on their carrier types. Then, the decomposition voltage is written as below,

\section{Decomposition Voltage $=$ \\ Overvoltages (anode and cathode) $+\mathrm{E}$}

It is clear that the decomposition voltage is the minimum voltage for the electrolysis of water. A conditional decomposition voltage obtained from the experiments is considered to be a larger value. An AFM system (SPI-400 of SII nano technology Inc.) was used for the anodization. The canti-lever of the AFM was

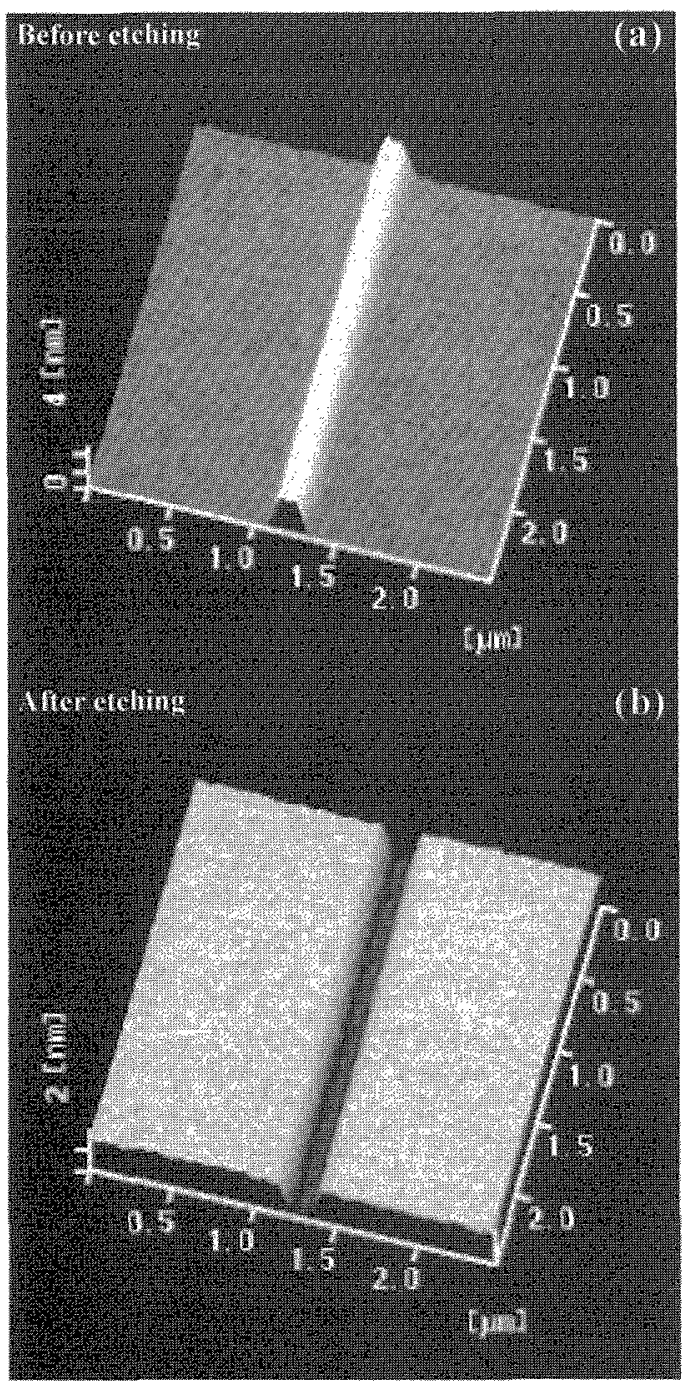

Fig. 2. $\mathrm{A} \mathrm{SiO}_{2}$ single line formed on a p-Si (100) surface by an atomic force microscope induced anodization. (a) as-anodized and (b) after etching the oxide in a dilute HF solution.

uniquely biased as cathode. In contrast, a plus voltage was applied on the $\mathrm{Si}$ wafer as the anode. The canti-lever was scanned or spotted on the Si surface to from line and point oxides, respectively. A $\mathrm{He}-\mathrm{Ne}$ laser was used to measure the tip position during the scan. This light having energy of $1.8 \mathrm{eV}$ may generate additional electron-hole $(\mathrm{e}-\mathrm{h})$ pairs in $\mathrm{Si}$ during the anodization. After the anodization the size and shape of the oxides were measured by the same AFM. On the other hand, the groove and pits after etching the sample in a dilute HF solution were measured as the same manner, too.

\section{Results and discussion}

Shown in Fig. 2 are the AFM images of a single oxide line formed on $\mathrm{p}-\mathrm{Si}(100)$ surface. The left picture shows the as-anodized image. A $2.5 \mathrm{~mm}$ long line with height of $4 \mathrm{~nm}$ and width of $200 \mathrm{~nm}$ was obtained by 
one scan of the tip under $9 \mathrm{~V}$ applied bias voltage. The line was along the tip scanning trace and its height and width were uniform, indicates the capability of the AFM induced anodization. The right part of Fig. 2 shows the groove formed by etching the anodized wafer in a dilute HF solution. An oxidizing depth of $2 \mathrm{~nm}$ was obtained. Although the groove might be deeper due to the large curvature radius of the $\mathrm{Rh}$-coated AFM tip, the uniform grooves were fabricated by the anodization, too.

The applied bias voltage dependence of the average line width of the oxide is shown in Fig. 3(a). Three types of $\mathrm{Si}(100)$ wafers, $\mathrm{p}, \mathrm{n}$ and high resistive (HR), were used. It was shown that the anodization began at different bias voltages for different carrier types. The p-Si started oxidizing at the applied bias of $2.2 \mathrm{~V}$. In contrast, $3.6 \mathrm{~V}$ and $4.3 \mathrm{~V}$ were obtained for the HR and n-type Si samples, respectively; This difference first comes from the Fermi level difference of the samples, because the anodization is a hole assisted process. It is necessary to generate holes in the $\mathrm{Si}$ surface by increasing bias voltage for the cases of HR and n-type wafers. The Fermi level difference between samples was about $0.5 \mathrm{~V}$ each for $\mathrm{p}$ and $\mathrm{HR}, \mathrm{HR}$ and $\mathrm{n}-\mathrm{Si}$, respectively. The starting bias voltages of the anodization for each case were considered to change by $0.5 \mathrm{~V}$ and in the order of $\mathrm{p}<\mathrm{HR}<\mathrm{n}$. The ordering was as our prediction, but the starting voltages showed larger values. This difference might come from the different overvoltages caused by the different carrier types, and also come from the series resistance of the samples having higher resistivity. The hole in n-type $\mathrm{Si}$ is the minority carrier, the hole-rich surface needs higher applied bias which would increase the width of the depletion layer and increase the series resistance. Shown in Fig. 3(b) is the applied bias voltage dependence of the average oxide height for the three type Si wafers. A similar discussion can be deduced like the case of the line width. The height of the oxide lines increase almost linearly with increasing bias voltage. A similar manner can be seen in the anodization of $\mathrm{Si}$ using the purified water as the electrolyte. A narrower and higher oxide line is relatively formed by using the n-type $\mathrm{Si}(100)$ wafer. The applied bias voltage dependence of the groove after etching the oxide line almost as same as the data shown in Fig. 3. We will omit it here due to the paper length limit.

Figure 4 shows pointing time dependence of the average diameter of the spot oxide formed on $\mathrm{p}-\mathrm{Si}(100)$ surface by AFM induced anodization. The inset shows a single oxide dot fabricated by applying bias of $2 \mathrm{~V}$ and pointing time of $10 \mathrm{~s}$, respectively. The oxide diameter increased linearly with the increasing pointing time. The oxide height also showed the similar pointing time dependence. The spot type oxidization would be useful for forming insulator islands in nanodevice application.

\section{Conclusions}

We have fabricated line and spot type $\mathrm{SiO}_{2}$ regions on Si (100) surface by using an AFM induced anodization. The average width and height of the oxide lines depend on the applied bias voltage, humidity of the sample surface and carrier type. The difference of the starting voltage of oxidization for different carrier type $\mathrm{Si}$ wafers
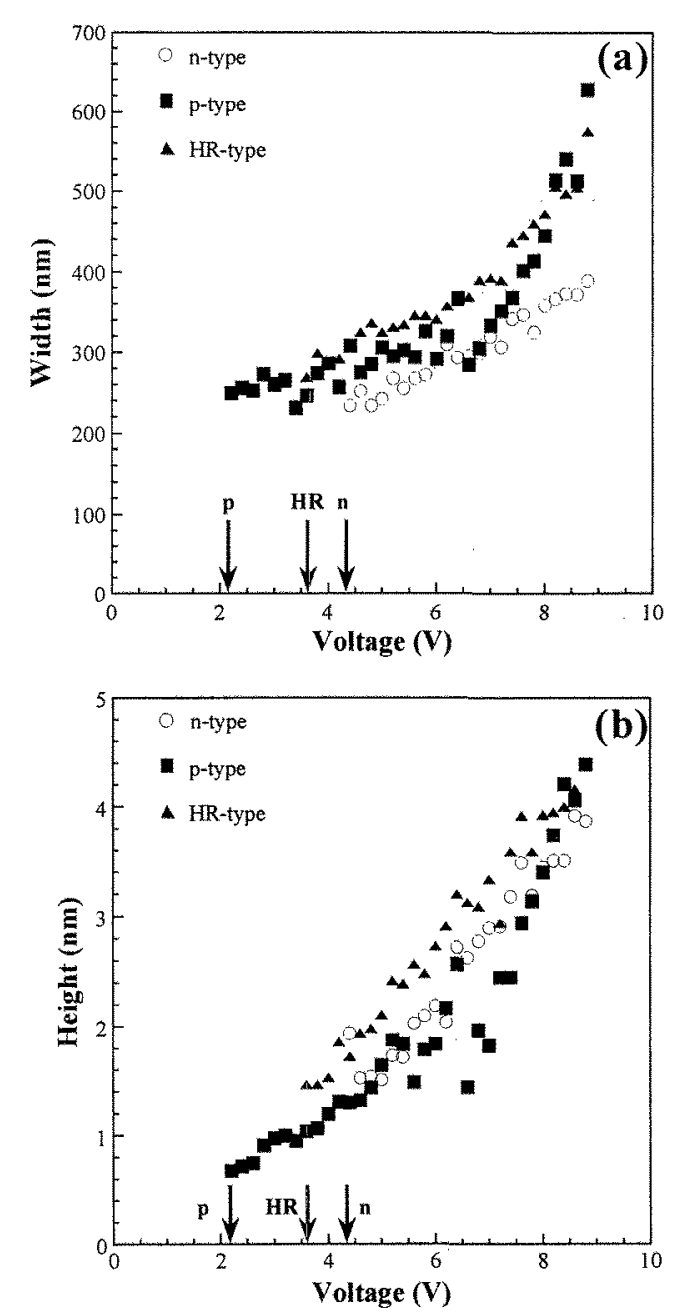

Fig. 3. The applied bias voltage dependence of the line width (a) and the line height (b) of the line type oxide formed on p, HR and n-type Si (100) surfaces by an atomic force microscope induced anodization.

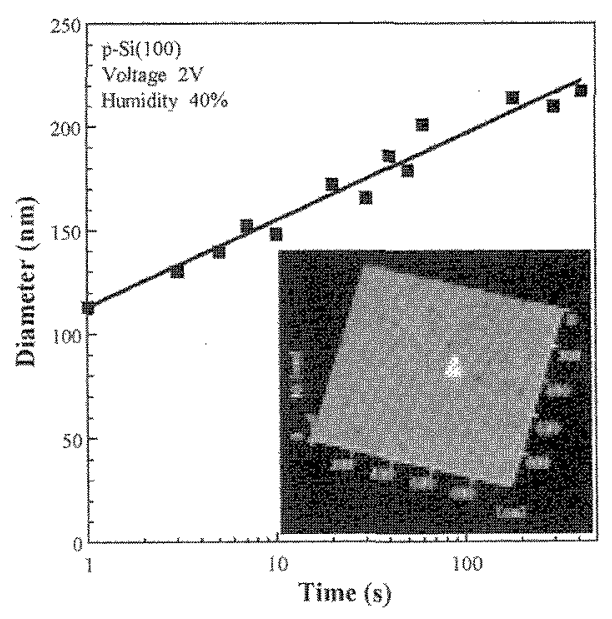

Fig. 4. The pointing time dependence of the spot type oxide formed on a p-type Si (100) surface by an atomic force microscope induced anodization. The inset shows a single $\mathrm{SiO}_{2}$ dot formed on the p-Si surface. 
indicates a hole assisted anodization process. Narrow and deep lines have been obtained for the case of $\mathrm{n}-\mathrm{Si}$. Our works show the possibility of AFM anodization in nanofabrication process.

We would like to thank Prof. K. Ohkawa and Prof. S. Ando for stimulating discussions and to thank Mr. T. Shirakawa for technical assistance. This work was partially supported by the Nanotech and Green Photonic Programs of Tokyo University of Science.

\section{References}

[1] G. Binnig, C.F. Quate, and Ch. Gerber, Phys. Rev. Lett., 56, 930 (1986).

[2] J.A. Dagata, J. Schneir, H.H. Harary, C.J. Evans, M.T.Postek, and J. Benett, Appl. Phys. Lett., 56, 2001 (1990).

[3] Ph. Avouris, R. Martel, T.Hertel, and R. Sandstrom, Appl. Phys. A, Mater. Sci. \& Proc., A 66, S659 (1998).

[4] K. Matsumoto, M. Ishii, K. Segawa, Y. Oka, B. J. Vartanian, and J. S. Harris, Appl. Phys. Lett., 68, 34 (1996).

[5] K. Matsumoto, Y. Gotoh, T. Maeda, J. A. Dagata, and J. S. Harris, Appl. Phys. Lett., 76, 239 (2000).

[6] H. Dai and N. Franklin, and J. Han, Appl. Phys. Lett., $73,1508(1998)$.

[7] H. Kuramochi, K. Ando, and H. Yokoyama, Surf. Sci. 542,56 (2003).

[8] K. Ueno, R. Okada, K. Saiki, and A. Koma, Surf. Sci. 514, 27 (2002).

[9] F. Pérez-Murano, K. Birkelund, K. Morimoto, and J. A. Dagata, Appl. Phys. Lett., 75, 199 (1999).

[10] B. E. Conway, and J. O'M. Bockris, J. Chem. Phys., 26,532 (1957).

[11] H. J. Lewerenz, Elctrochimica Acta, 37, 847 (1992).

[12] V. I. Veselovsky, T. I. Borisova, A. A. Jakovleva and S. O. Izidinov, Elctrochimica Acta, 10, 315 (1965). 